\section{Vasculitis of the Female Genital Tract: Not Always a Localized Condition}

\section{To the Editor:}

Giant cell arteritis (GCA) is the most common systemic vasculitis in adults, with a European and North American prevalence of $0.2 \%$, increasing with northern latitude ${ }^{1,2}$. In contrast, gynecological vasculitis is rare ${ }^{3}$, with the first case described by Plauth in $1932^{4}$. Cases are divided into localized and systemic disease. Where a systemic disorder can be identified, GCA is the most common diagnosis ${ }^{3}$.

A 79-year-old European woman presented to her general practitioner complaining of fatigue, malaise, and discomfort along the left side of her abdomen, with $2 \mathrm{~kg}$ weight loss. This progressed to colicky right upper-quadrant pain. A laparoscopic cholecystectomy revealed a thin-walled gall bladder with a retention mucocele. Her symptoms did not resolve and an ultrasound scan revealed a pelvic mass adjacent to the endometrium roughly $150 \times 100 \times 70 \mathrm{~mm}$. She denied any postmenopausal bleeding and there was nothing palpable on abdominal examination. She underwent an open hysterectomy and bilateral salpingoophrectomy.

Histology revealed the pelvic mass was an edematous uterine intramural submucous myoma with no evidence of malignancy. The uterus and fallopian tubes showed foci of vasculitis involving arterial vessels, with features including medial destruction by a lymphocytic and histiocytic infiltration, features consistent with GCA (Figure 1).

Investigations revealed C-reactive protein (CRP) $2 \mathrm{mg} / \mathrm{l}$, erythrocyte sedimentation rate (ESR) $6 \mathrm{~mm} / \mathrm{h}$, and hemoglobin $(\mathrm{Hb}) 13.9 \mathrm{~g} / \mathrm{dl}$. She denied any headache, jaw claudication, general malaise, significant weight loss, or double vision. Thus, there was no clinical or laboratory evidence of active systemic vasculitis and she was not treated with steroid or other immunosuppressive therapy.

Twelve months later, she presented again with nonspecific symptoms, with rising inflammatory markers (CRP $63 \mathrm{mg} / \mathrm{l})$ and falling $\mathrm{Hb}(10.6$ $\mathrm{g} / \mathrm{dl}$ ). Investigations including blood and urine cultures and computed tomography scanning of thorax, abdomen, and pelvis did not reveal infective or neoplastic evidence. She developed a chronic headache and generalized myalgia, and a temporal artery biopsy was performed. This showed active GCA (Figure 2). She was started on high-dose oral corticosteroids 1 $\mathrm{mg} / \mathrm{kg}$; symptoms settled rapidly with normalization of CRP and $\mathrm{Hb}$. Her

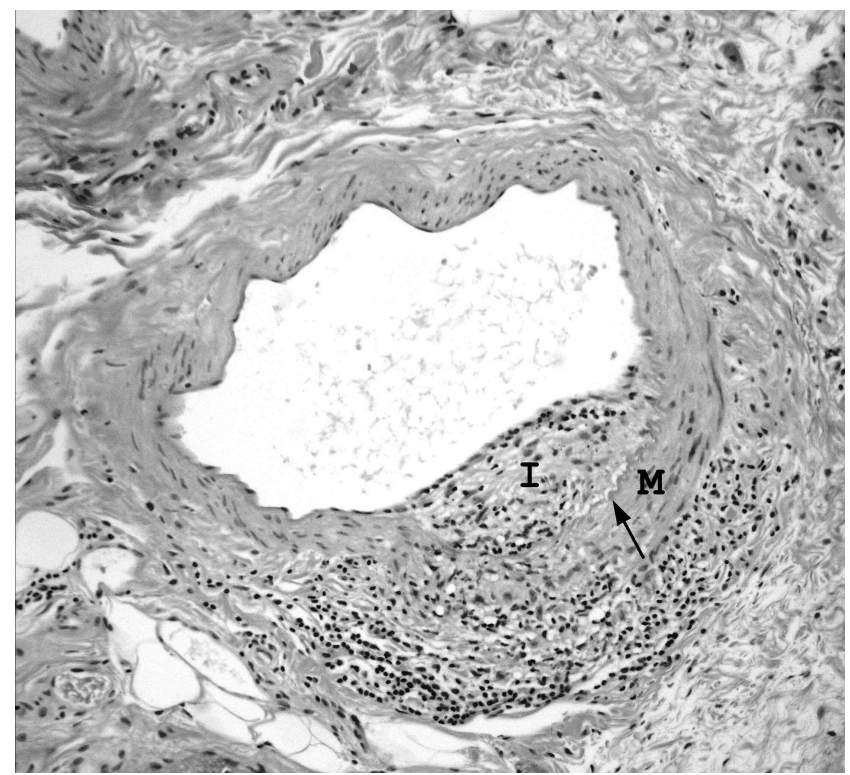

Figure 1. Artery in tubal mesentery showing lymphohistiocytic infiltrate damaging media (M), extending to intima (I), with intact intervening internal elastic lamina (arrow).

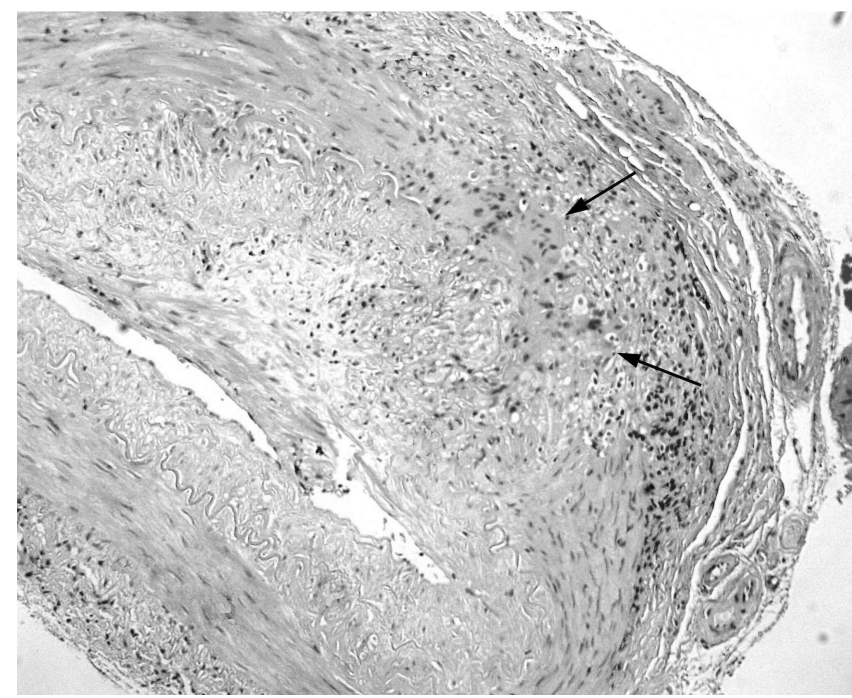

Figure 2. Temporal artery showing transmural lymphohistiocytic infiltrate with giant cells (arrows).

steroid dose was successfully tapered with no recurrence of symptoms. At last followup 8 months after starting corticosteroids, she was receiving prednisone $12.5 \mathrm{mg} / \mathrm{day}$, along with appropriate bone prophylaxis. She has had no recurrence of symptoms and $\mathrm{Hb}$ and $\mathrm{CRP}$ are within the normal range.

Medium to large vessel gynecological vasculitis is rare ${ }^{3,5}$, but often presents with common gynecological signs and symptoms including vaginal bleeding, uterine prolapse, asymptomatic abdominal mass, and pelvic pain. The majority of cases are organ-limited, but when the systemic form is present this most commonly has features of GCA. Given that the reproductive tract is not a typical place for vasculitis, diagnosis is often delayed.

Localized vasculitis is typically benign with a good prognosis ${ }^{3}$. However, Piura, et al reviewed 30 cases reported up to $2004^{6}$ and concluded that the incidental finding of GCA on gynecological specimens should raise suspicion of systemic involvement, prompting further clinical and laboratory scrutiny, as symptoms may be elicited on directed questioning. Hernandez-Rodriguez, $e t a l^{3}$ reported an incidence of gynecological vasculitis over 16 years at $0.15 \%$, with up to half of these presenting with the common gynecological complaints. Just over $70 \%$ had isolated gynecological vasculitis. From their series of 163 patients a normal ESR and absence of anemia or systemic symptoms suggested organ-limited disease and systemic therapy was not required; however, this did not preclude the diagnosis of systemic vasculitis.

Orbo and Steffensen ${ }^{7}$ described a case of uterine GCA in a histological specimen removed for low-risk endometrial carcinoma. There were no symptoms or signs of systemic vasculitis in the perioperative period. Temporal artery biopsies were performed in the absence of symptoms and were positive. This suggests that histological findings of localized vasculitis can predate symptoms and signs of systemic disease, and close clinical followup of these patients is warranted.

We describe a patient who presented with localized gynecological vasculitis and was later diagnosed with temporal arteritis. This could represent the progression of a localized into a systemic vasculitic process. This is rare for gynecological vasculitis, which is usually regarded as benign and not requiring systemic therapy, but evolution into GCA has been described $^{3}$. A definitive diagnosis of single-organ vasculitis can only be considered after a followup period long enough to define it as an isolated process. In this case, features of GCA developed after 1 year. Alternatively, our case could represent a situation where systemic GCA was already present but was asymptomatic with regard to the classical symptoms of temporal arteritis, the presentation being dominated by gynecological manifesta-

Personal non-commercial use only. The Journal of Rheumatology Copyright @ @ 2012. All rights reserved. 
tions. We cannot exclude this, as temporal artery biopsy was not performed at the initial presentation. Whichever scenario is correct, it indicates that patients with apparently localized vasculitis should be followed clinically for at least 12 months. The development of suggestive symptoms or rising inflammatory markers should prompt further investigation, which may include a temporal artery biopsy.

STEPHEN C. WEST, MBBS, Medical Registrar, Department of General Medicine; L. JONATHAN ZWI, FRCPA, Consultant, Histopathologist, Histopathology Laboratory; DAVID SPRIGGS, MD, Consultant, General Physician, Department of General Medicine; FIONA M. McQUEEN, MD, Professor of Rheumatology, Department of Rheumatology, Auckland City Hospital, Auckland District Health Board, Auckland, New Zealand. Address correspondence to Dr. West, Auckland City Hospital, General Medicine, 2 Park Road Grafton, Auckland 1023, New Zealand;

E-mail: stephenwest@doctors.org.uk

We thank Dr. Kai Yin Chau and Dr. Noelyn Gordon-Glassford for providing access to histological samples.

\section{REFERENCES}

1. Richards BL, March L, Gabriel SE. Epidemiology of large-vessel vasculidities. Best Pract Res Clin Rheumatol 2010;24:871-83.

2. Weyand CM, Goronzy JJ. Medium- and large-vessel vasculitis. N Engl J Med 2003;349:160-9.

3. Hernandez-Rodriguez J, Tan CD, Rodriguez ER, Hoffman GS Gynecologic vasculitis: An analysis of 163 patients. Medicine 2009;88:169-81

4. Plauth A. Focal arteriolitis. Am J Pathol 1932;8:620-1.

5. Hoen B, Baty V, Aymard B, Judlin P, Foliguet B, Canton P. Giant-cell arteritis of the female genital tract. J Intern Med 1994;236:345-7.

6. Piura B, Wolak A, Rabinovich A, Yanai-Inbar I. Giant cell arteritis of the female genital tract. Clin Exp Obstet Gynecol 2004;31:31-3.

7. Orbo A, Steffensen A. Endometrial cancer, vasculitis of the genital tract and occult temporal arteritis. Histopathology 2001;38:178-9.

J Rheumatol 2012;39:11; doi:10.3899/jrheum.120795 